\title{
Penser théologiquement les droits de l'homme: entre universalité, historicité et pluralité
}

\author{
Pensar teologicamente os direitos humanos: \\ entre universalidade, historicidade e pluralidade
}

\section{Joël Molinario*}

Institut Catholique de Paris, Paris, France

\begin{abstract}
Les droits de l'homme sont entrés dans une période d'extension et de doute. D'extension, parce que depuis la déclaration universelle des droits de l'homme de 1948, d'autres déclarations onusiennes ont été proclamées, mais aussi de doute parce les droits de l'homme ne peuvent échapper à leur origine occidentale qui fait douter certains de leur universalité. Pour un théologien catholique, penser théologiquement les droits de l'homme n'est pas aisé pour une raison historique qui tient au rejet radical dont ceux-ci ont été l'objet par le Magistère catholique au XIXè siècle, comparé au changement de doctrine dans la seconde moitié du XX⿳亠㐅八 siècle. Cet article mène une enquête historique, philosophique et théologique sur les droits de l'homme et traverse quelques questions essentielles pour la théologie aujourd'hui : celle de l'historicité des opinions et des doctrines, celle de l'universalité du salut, des principes et des pratiques et celle
\end{abstract}

*JM: Docteur, e-mail: jmolinario@icp.fr 
de la pluralité radicale des fondements sur lesquelles bâtir un bien commun. II apparait que l'exigence de travailler théologiquement les droits de l'homme touche le cœur de la Révélation la rencontre de l'altérité au nom de l'universalité du salut.

Mots clés : Droits de l'homme. Liberté. Historicité. Philosophie. Universalité du salut.

\section{Resumo}

Os direitos humanos adentraram em um período de extensão e dúvida. De extensão, porque desde a Declaração Universal dos Direitos Humanos, de 1948, outras declarações da ONU foram proclamadas ; mas também de dúvida, porque os direitos humanos não podem escapar à sua origem ocidental, o que faz com que alguns duvidem de sua universalidade. Para um teólogo católico, pensar teologicamente os direitos humanos não é fácil por uma razão histórica que consiste na rejeição radical da qual eles foram objeto pelo Magistério católico no século XIX, comparativamente à mudança de doutrina na segunda metade do século XX. Esse artigo empreende uma investigação histórica, filosófica e teológica sobre os direitos humanos e atravessa algumas questões essenciais para a teologia atual : a historicidade das opiniões e doutrinas, a universalidade da salvação, dos princípios e das práticas e a pluralidade radical dos fundamentos sobre os quais se busca construir um bem comum. Ocorre que a exigência de trabalhar teologicamente os direitos humanos toca no coração da Revelação, o encontro da alteridade em nome da universalidade da salvação.

Palavras-chave: Direitos humanos. Liberdade. Historicidade. Filosofia. Universalidade da salvação.

\section{Introduction}

Les droits de l'homme sont entrés dans une période à la fois d'extension et de doute. D'extension, parce que depuis la déclaration universelle des droits de l'homme de 1948, d'autres déclarations 
onusiennes ont été proclamées, jusqu'à des droits écologiques, mais aussi de doute parce les droits de l'homme ne peuvent échapper à leur origine occidentale qui fait douter certains de leur universalité et d'autres les refuser purement et simplement. Cette remise en cause n'est pas seulement l'œuvre de dictateurs sanguinaires, mais aussi de penseurs respectables réinterrogeant leurs légitimités. Pour un théologien catholique, penser théologiquement les droits de l'homme n'est pas aisé non plus pour une première raison historique qui tient au rejet radical dont ceux-ci ont été l'objet par le Magistère catholique au XIX ${ }^{e ̀}$ siècle, comparé au revirement à 180 degrés des papes dans la seconde moitié du XXè siècle. Alors est-il possible de penser théologiquement les droits de l'homme et comment les comprendre aujourd'hui, quels sens et quel avenir représentent-ils pour les sociétés et quel en est l'enjeu théologique? De pareils aléas historiques et des changements de doctrines théologiques autant que philosophiques et politiques à leurs sujets ainsi que la grande pluralité d'interprétations contemporaines n'invalident-ils pas toute tentative sérieuse de penser théologiquement les droits de l'homme?

Les publications étant abondantes sur le sujet, il faudra faire des choix sans pour autant éviter de placer en perspective historique et politique les droits de l'homme, au moins depuis la fin XVIII è siècle, puis discerner la justesse et les enjeux anthropologiques et théologiques des débats actuels à leurs sujets qui nous fera rencontrer la question de la radicale pluralité des anthropologies et des fondements religieux possibles de tels droits et le doute sur une universalité dont l'origine occidentale est devenu un problème pour les autres cultures. Dans cette exploration des droits de l'homme nous traverserons par un parcours obligé, les questions de l'historicité, de l'universalité et de la pluralité. Nous pourrons ainsi vérifier s'il s'agit bien d'une question qui concerne la théologie chrétienne et si les chrétiens doivent s'y engager.

\section{Trois déclarations fondatrices et les liens entre elles}

Trois textes historiques ont mis à jour la notion moderne de droits de l'homme. Il s'agit de la Déclaration d'indépendance américaine 
de 1776, de la Déclaration des droits de l'homme et du citoyen (DDHC) de la constituante du 26 août 1789 et enfin et surtout de la Déclaration universelle des droits de l'homme (DUDH) proclamée par l'ONU le 10 décembre 1948. Chacune de ces déclarations ayant engendré d'autres déclarations. La DDHC a été suivie d'une seconde déclaration émanant de la constituante en juin 1793, plus longue, elle comporte 35 articles, alors que la DDHC n'en comporte que 17. La DUDH ne fut pas non plus une fin en soi, mais engendra la déclaration universelle sur le droit des enfants de 1959 ainsi que la déclaration sur les droits civiques de 1966 et d'autres plus récentes sur les droits liés à l'écologie. Le lien de la DUDH vis-à-vis à de la déclaration de la Constituante de 1789 est assez facile à montrer, par la forme, par la manière de rédiger ainsi que par des phrases reprises avec une évidente inspiration. Dans les articles premiers « Les hommes naissent libres et égaux en droits » (DDHC art.1) ; "Tous les êtres humains naissent libres et égaux en dignité et en droits » (DUDH art.1), le parallèle s'impose. Dans l'article 11 de la DUDH nous lisons "Toute personne accusée d'un acte délictueux est présumée innocente », inspiration directe de l'article 9 de la DDHC qui dit : " Tout homme étant présumé innocent jusqu'à ce qu'il ait été désigné coupable... ». De même, les notions de liberté, d'égalité et de fraternité de l'article premier de la DUDH sont une évocation de la Révolution française (même si le mot fraternité n'est pas présent dans la DDHC) et que ce ternaire ne sera la devise française qu'en 1848. Cependant, il n'y a pas d'exclusivité inspiratrice de la DDHC. La déclaration d'indépendance de 1776 exprime déjà l'idée de liberté comme droit inaliénable, et l'égalité comme principe de l'homme créé. De même, dans la Constitution du Massachusetts de 1780 nous trouvons les mots libertés et égalité accolés : "All men are born free and equal. » Nous connaissons par ailleurs des liens entre les déclarations américaines et révolutionnaires françaises par la personne du marquis de Lafayette qui, convaincu du bienfondé des déclarations américaines, proposa deux versions de déclarations des droits de l'homme en juillet 1789 . Elles ne furent pas retenues mais l'idée d'une déclaration fit son chemin dans la tête des députés de la Constituante et le débat serré qui eut lieu en août 1789 autour de la conception de la liberté est le signe du lien entre les déclarations américaines et françaises. Mirabeau s'en fit l'ardent défenseur avec une phrase restée célèbre : «Je 
ne viens pas prêcher la tolérance. La liberté la plus illimitée de religion est à mes yeux un droit si sacré, dit-il, que le mot tolérance, qui essaye de l'exprimer me parait lui-même tyrannique, puisque l'existence de l'autorité qui a le pouvoir de tolérer, attente à la liberté de penser, par cela même qu'elle tolère, et qu'ainsi elle pourrait ne pas tolérer. » $^{1}$

\section{Réceptions de la notion de droits de l'homme chez les catholiques, de l'opposition au soutient}

Le lien entre les droits de l'homme et Eglise catholique a toujours été un sujet sensible, en France tout spécialement. Hormis, l'étude historique particulièrement documentée de Valentine Zuber², le contexte idéologique et politique français n'a pas permis sereinement de travailler la possible inspiration religieuse des droits de l'homme. Longtemps, les postures anticléricales d'un côté, catholique intransigeante de l'autre ont empêché toute réflexion historique, juridique, philosophique et théologique. Avec un curieux rapprochement entreles deux positions, puisqueles jacobins criaient victoire, «la république qui s'est libérée du pape a permis la Déclaration des droits de l'homme et du citoyen » et de l'autre, les intransigeants validant l'appropriation républicaine jacobine justifiant d'autant plus ainsi leur opposition aux droits de l'homme et à l'impensable liberté religieuse.

Pourtant, la question religieuse était présente dès le début : dans les déclarations étasuniennes de 1776, mais aussi dans l'article 10 de la DDHC du 26 août 1789 qui proclame que : « Nul ne peut être inquiété pour ses opinions, même religieuse, pourvu que sa manifestation ne trouble pas l'ordre public ». L'intervention brillante de Mirabeau défendant cette liberté religieuse semble avoir emporté la partie face aux jacobins et aux conservateurs du clergé.

1 Valentine ZUBER, L'origine religieuse des droits de l'homme, le christianisme face aux libertés modernes (XVIIIè-XXlè siècle), Genève, Labor et Fides, 2017, p.19, l'auteur fait référence aux Archives parlementaires rééditées régulièrement et accessibles aujourd'hui sur Gallica.

2 Ibid. L'origine religieuse des droits de l'homme, Genève, Labor et Fides, 2017. 
Cette même liberté sera qualifiée d'inaliénable par la Déclaration universelle des droits de l'homme de l'ONU et fit l'objet des quatre premiers articles du texte proclamé le 10 décembre 1948.

A Vatican II, après d'interminables débats, le Concile proclama que : « Le droit à la liberté religieuse a son fondement réel dans la dignité même de la personne humaine telle que l'ont fait connaître la Parole de Dieu et la raison elle-même. Ce droit de la personne humaine à la liberté religieuse dans l'ordre juridique de la société doit être reconnu de telle manière qu'il constitue un droit civil ${ }^{3}$ ». Cette citation provient de Dignitatis Humanae personae, (DH) qui est une déclaration selon la terminologie du concile, c'est-à-dire que Vatican II reprit le terme utilisé par l'ONU et ceci ne peut être un hasard, mais bien le fait, comme l'explique le Père Congar, que ce texte s'adresse à tous les hommes de bonne volonté et pas seulement aux fidèles. ${ }^{4}$ Nous connaissons l'histoire tourmentée de ce texte et le difficile travail des commissions et comment il a été combattu par la minorité conciliaire jusqu'au bout. Les épisodes de la crise d'octobre 1964 et de la semaine noire de novembre de cette même session du concile en témoignent, qui vont occasionner malgré l'opposition d'une grande majorité le report du vote définitif du texte à la dernière session. ${ }^{5}$ Les divergences fondamentales entre la commission doctrinale et le secrétariat pour l'unité de chrétiens ont dû plus d'une fois, faute d'accord, être arbitrées par Paul VI. De ce conflit dans la rédaction, il en sortit un compromis où les fondements bibliques et théologiques ont été esquivés au profit d'un axe nettement politique et juridique. ${ }^{6}$ Ce qui permet, cependant, un rapprochement avec la DUDH de 1948, et le paragraphe 15 de DH est explicite : «la liberté religieuse est maintenant proclamée dans la plupart des Constitutions comme un droit civil et qu'elle est solennellement reconnue par des documents internationaux. " ${ }^{7}$

3 Dignitatis Humanae personae, 7 décembre 1965, $\mathrm{n}^{\circ} 2$.

4 Yves M-J. Congar, "Que faut-il entendre par déclaration ? " dans Vatican II, la liberté religieuse, Paris, collection Unam Sanctam, éditions du Cerf, 1967, p.47-52.

5 Giuseppe Alberigo, Histoire du Concile Vatican II, 1959-1965, Tome IV, I'Eglise en tant que communion, Louvain/ Paris, Cerf/Peeters, 2003, chapitre II et chapitre VI.

6 Giuseppe Alberigo, Histoire du Concile Vatican II, 1959-1965, Tome IV, I'Eglise en tant que communion, Louvain/ Paris, Cerf/Peeters, 2003, chapitre II et chapitre VI.

7 Dignitatis humanae personae, $\mathrm{n}^{\circ} 15$. 
Ces débats très durs au concile procédaient d'une longue histoire. Après les déclarations d'indépendance américaine et la Déclaration des droits de l'homme et du citoyen, l'Eglise catholique s'est prononcée officiellement et violemment contre la liberté religieuse et ce ci de façon répétée tout au long du XIXe siècle et jusqu'au seuil du Concile Vatican II. Le pape Grégoire XVI en 1832 qualifia la liberté proclamée par les droits de l'homme de "maxime fausse ", " absurde ", " extravagante ", "d'erreur des plus contagieuse », " monstrueuse doctrine », " pour la ruine de l'Eglise et de l'état ${ }^{8}$. Ce ne fut pas le cas des Eglises protestantes, qui non seulement accompagnèrent mais d'une certaine manière anticipèrent la formulation et la proclamation des droits et notamment par le biais de la liberté religieuse comme le montra déjà Georg Jellinek au tout début du XX ${ }^{\text {siècle. }}{ }^{9}$

Cette franche évolution de la doctrine de l'Eglise au sujet des droits de l'homme est une précieuse indication à la fois pour comprendre la Déclaration universelle des droits de l'homme mais aussi pour comprendre comment la foi de l'Eglise évolue et mûrit dans son tissage avec l'histoire des hommes.

\section{L'impossible consensus sur les fondements philosophiques et théologiques. L'UNESCO, Jacques Maritain et la pluralité des fondements}

A l'UNESCO fut confié la discrète mais redoutable tâche de justifier rationnellement et philosophiquement ces droits valables pour tous les hommes de toutes les nations et de toutes les cultures. En vue de la préparation de la DUDH de 1948, L'UNESCO donna mission à plusieurs personnalités, dont le philosophe français Jacques Maritain d'écrire un rapport qui permettrait de justifier ces droits de l'homme universels. Les

8 Mirari vos Grégoire XVI 15 août 1832.

9 La littérature est abondante sur le sujet : Georg JELLINEK, La déclaration des droits de l'homme et du citoyen : contribution à l'étude du droit constitutionnel moderne, traduction de l'allemand, Paris, Fontemoing,1902 ; Hans JOAS, Comment la personne est devenue sacrée, nouvelle généalogie des droits de l'homme, traduit de l'allemand par Jean-Marc Tétaz, Genève, le champ éthique, Labor et Fides, 2015 ; Valentine ZUBER L'origine religieuse des droits de l'homme, le christianisme face aux libertés modernes (XVIIIè-XXIè siècle), Genève, Labor et Fides, 2017. 
auteurs du rapport s'en expliquent ainsi : «Une déclaration internationale des Droits de l'homme doit être l'expression d'une foi à tenir, pas moins qu'un programme d'actions à mener. C'est un fondement pour des convictions universellement partagées par les hommes, quelles que soient l'ampleur des différences de situation et de manière de formuler les Droits de l'homme, c'est un élément essentiel dans la structure constitutionnelle des Nations Unies. ${ }^{10}$ » Les auteurs poursuivent ainsi, "Un examen des fondements diune charte des droits devrait donc servir à révéler, d`une part, les principes communs sur lesquels repose la déclaration et à anticiper, drautre part, certaines des difficultés et des différences d'interprétation... ${ }^{11}$

Le comité de préparation de ce rapport demanda à 70 personnalités du monde entier de livrer leur avis par écrit sur la justification d'une pareille déclaration universelle des droits de l'homme. Ainsi fut constitué un dossier de près de 300 pages émanant de philosophes, de scientifiques, d'économistes, d'hommes politique, de religieux, de théologiens de tous les continents et de toutes opinions ${ }^{12}$. Le dossier débute par une touchante lettre du Mahamat Gandhi et se termine par le texte du britannique Margery Fry secrétaire général de la ligue « for the Penal Reform. ${ }^{13}$ "

Mais ce projet idéal de justification se heurta à la réalité de la pluralité des systèmes philosophiques, des anthropologies, des religions, des disciplines scientifiques, des convictions de toutes sortes et des traditions culturelles, si bien qu'en introduction Jacques Maritain reconnut l'impossibilité théorique d'un tel projet qui rend compte de la pluralité extrême des opinions mais qui ne permet nullement de tenir une synthèse consensuelle. Jacques Maritain rapporta l'anecdote suivante qui résume l'échec du projet de justification commune : au cours d'une « des réunions d'une commission nationale de l'Unesco où l'on discutait des droits de l'homme, quelqu'un s'étonnait que certains défenseurs d'idéologies violemment opposées se soient mis d'accord sur une liste de ces droits.

\footnotetext{
10 The ground of an international declaration of Human Rights25 July 1948, Appendix II. Traduction personnelle. 11 Ibid.

12 UNESCO/PHS/3 rev. Paris, July 1948.

${ }^{13}$ La plus ancienne organisation au monde pour la défense du droit des prisonniers et la lutte contre la peine capitale.
} 
«Oui», ont-ils dit, «nous sommes d'accord sur les droits mais à condition que personne ne nous demande pourquoi. ${ }^{14}$ " Le paradoxe, explique Maritain, est que de telles justifications rationnelles sont à la fois indispensables, et pourtant impuissantes à amener un accord entre les esprits.

En somme, un tel projet correspondant à une conception descendante et globalisante d'un principe d'universalité, qui exposerait ce qu'est l'être humain d'où l'ONU pourrait déduire des droits humains, fut sévèrement battu en brèche non par les tyrans sanguinaires que l'on vit agir durant la seconde guerre mondiale, - et qui n'accordent aucun crédit à une déclaration des droits de l'hommes-, mais par des hommes raisonnables et de bonne volonté. Cette manière de penser l'universel, comme une manière qui s'impose à tous de penser pareillement fut déjà, en 1948, considérée comme intenable.

Dès 1948 était donc posé à nouveau la question complexe de l'universalité à propos des droits de l'homme mais en réalité c'est bien la question de l'universalité de l'humain dont il était question puisque sur les droits il se fit un accord. Comment vivre ensemble, pourquoi défendre la vie humaine et les droits des individus et des peuples si l'on n'est pas capable de partager un consensus humaniste ? Alors il serait toujours possible de justifier toutes les barbaries du monde ? Pourtant, la pluralité des conceptions de l'homme est une réalité qu'on ne peut que constater, (réalité gênante puisque l'UNESCO ne publia jamais ce rapport) mais l'enjeu est bien de savoir si cette pluralité est une valeur que l'on reconnait, c'est-à-dire un pluralisme? Et ainsi, sur quel type d'universalité peut-on s'accorder?

\section{Jean-Paul II et le changement de doctrine dans le discours à I'UNESCO : les droits comme fondements culturels}

Après Jean XXXIII, dans Pacem in Terris, ${ }^{15}$ et le concile Vatican II, JeanPaul II ira plus loin sur les droits de l'homme, dans son discours prononcé

\footnotetext{
14 "Yes", they said, "we agree about the rights but on condition that no one asks us why." UNESCO/PHS/3 rev. Paris, July 1948, Introduction Jacques Maritain

15 Lettre encyclique sur la paix entre toutes les nations, fondée sur la vérité, la justice, la charité et la liberté, 11 avril 1963.
} 
à l'UNESCO en 1980. "Il y a quand même - et je l'ai souligné dans mon discours à l'ONU en me référant à la Déclaration Universelle des droits de l'homme - une dimension fondamentale, qui est capable de bouleverser jusque dans leurs fondements les systèmes qui structurent l'ensemble de l'humanité et de libérer l'existence humaine, individuelle et collective, des menaces qui pèsent sur elle. Cette dimension fondamentale, c'est l'homme, l'homme dans son intégralité, l'homme qui vit en même temps dans la sphère des valeurs matérielles et dans celle des valeurs spirituelles. Le respect des droits inaliénables de la personne humaine est à la base de tout. " ( $\left.n^{\circ} 4\right)$. En effet, pour Jean-Paul II, les droits de l'hommes sont la base de la culture or c'est la culture qui humanise. Les droits ne sont pas une tolérance mais la base culturelle du bien commun et de la possible entente entre les peuples. "La signification essentielle de la culture consiste, selon ces paroles de saint Thomas d'Aquin, dans le fait qu'elle est une caractéristique de la vie humaine comme telle. L'homme vit d'une vie vraiment humaine grâce à la culture. La vie humaine est culture en ce sens aussi que l'homme se distingue et se différencie à travers elle de tout ce qui existe par ailleurs dans le monde visible : l'homme ne peut pas se passer de culture. " ( $\left.{ }^{\circ} 6\right)$.

En osant dire que les Droits de l'homme sont la base de tout, le pape Jean-Paul II indique deux choses essentielles. D'abord, la déclaration de 1948, n'est ni un constat ni une loi, - ce n'est pas vrai que les hommes naissent libre et égaux-, ce n'est pas non plus un impératif, le texte est au présent, mais pourtant la déclaration a ceci de précieux qu'elle est un bien culturel qui permet à des nations, des cultures, des idéologies les plus opposées d'avoir une base sur laquelle échanger. C'est donc aussi l'expérience de la rencontre de la pluralité radicale des cultures, des sagesses, des religions qui est en cause. Ensuite, le chemin qui va de Grégoire XVI à Jean-Paul II nous montre que l'expression de la foi de l'Eglise s'affine, mûrit, plongée qu'elle est dans les débats du monde et la rencontre de l'altérité. Pourquoi, alors que Saint Paul avait affirmé dans l'épitre aux Galates, « qu'il n'y a plus ni esclave, ni homme libre, ni homme, ni femme... » Gal 3,28, l'Eglise n'a-t-elle pas immédiatement proclamé les droits de l'homme? Avec le recul de l'histoire la réponse parait plus lisible : les droits de l'homme ainsi que la vie de foi de l'Eglise, ne sont pas l'application d'une théorie intemporelle mais l'émergence dans l'histoire 
d'une maturation de la foi et la prise de conscience d'un contour, certes encore imprécis, d'un bien commun universalisable qui peut voyager et évoluer entre les cultures. Nous retrouvons au niveau théologique un débat épistémologique et philosophique, dont les débattants sont d'un côté Kant et de l'autre Nietzsche. On ne peut, comme cela a été trop fait, opposer une universalité inconditionnelle et intemporelle, à une raison historique qui serait quant à elle forcément relativiste. L'historien et sociologue Hans Joas voit à travers cette histoire complexe des droits de l'homme un processus progressif de sacralisation de la personne à condition que l'on sorte du dilemme entre idéaux anhistoriques et relativité contingente de l'histoire ${ }^{16}$. «Le nom de Kant est associé à l'espoir de reprendre ou de développer une argumentation en philosophie morale qui soit capable de prétendre à une validité universelle et inconditionnelle indépendamment de toute historie. Le nom de Nietzsche marque la fin de tous les espoirs de ce genre, la prise de conscience du caractère irréductiblement subjectif de toute évaluation, mais aussi de la contingence de l'histoire, qui met aussi fin aux espoirs d'une fondation des valeurs recourant à la philosophie de l'histoire. ${ }^{17}$ » Entre les impasses kantienne et nietzschienne, il faut tracer une voie qui est celle d'une généalogie affirmative des droits de l'homme, qui tienne à la fois l'historicité et la continuité, les aléas contingents et l'affirmation de plus en plus reconnue des droits de l'homme. La thèse d'Hans Joas est stimulante et nous invite à penser la doctrine de l'Eglise dans cette histoire sans éviter les méandres, les débats, les contradictions que la prise au sérieux de l'histoire occasionne.

\section{Une opposition chrétienne aux droits de l'homme : Hauerwas, Simone Weil}

Plusieurs penseurs chrétiens ont soutenu que le langage des droits, bien que n'étant pas entièrement étranger au christianisme, est, dans

\footnotetext{
${ }^{16}$ Hans JOAS, Comment la personne est devenue sacrée, nouvelle généalogie des droits de l'homme, traduit de l'allemand par Jean-Marc Tétaz, Genève, le champ éthique, Labor et Fides, 2015, chapitre 4.

17 Ibid. Hans Joas, p.156.
} 
ses formes modernes, incompatible avec la foi et la pratique chrétienne. Le théologien Stanley Hauerwas affirme que les droits de l'homme sont conceptuellement et pratiquement inadéquats. Hauerwas reconnaît que "l'appel aux droits a été un moyen de protéger ceux qui n'ont aucune protection dans le monde dans lequel nous nous trouvons $» .{ }^{18}$ Il reconnaît en outre que de tels appels ont «fourni à beaucoup les éléments d'une identité morale autrement indisponible.» Pourtant, Hauerwas s'inquiète de ce que les « appels aux droits » réfléchis et répétés « menacent de remplacer les descriptions morales de premier ordre d'une manière qui nous rend moins aptes à rendre moralement sages les discriminations morales dont nous dépendons ». Par exemple, «Si vous avez besoin d'une théorie des droits pour savoir ... que la torture est moralement mauvaise», écrit Hauerwas, «alors quelque chose a clairement mal tourné avec votre sensibilité morale.» Hauerwas semble être le plus troublé par les droits qui autorisent les individus à choisir et à agir de manière socialement illimitée. Le droit à la liberté de "faire tout ce qui ne blesse personne, comme le disent les révolutionnaires français dans la DDHC est à la fois trop abstrait et trop diffus pour soutenir les engagements moraux et les jugements implicites dans la reconnaissance que toutes les personnes partagent une humanité commune. En Citant Esther Reed, Hauerwas affirme : «Aucune société réelle ne peut exister lorsque le seul lien entre ses citoyens est en termes de non-interférence » les uns avec les autres. En clair Hauerwas pense que les droits de l'homme sont une petite morale dont il ne faut pas se contenter et que la morale chrétienne est bien plus exigeante. ${ }^{19}$

La philosophe Simone Weil quant à elle, - qui pendant la seconde guerre mondiale s'opposa à Jacques Maritain sur le bien fondé d'une déclaration universelle des droits de l'homme-, se positionnait contre les droits, car pensait-elle, même si les droits peuvent jouer un rôle légitime dans les sphères juridiques, les droits ne sont ni moraux ni absolus, et nous détournent de la morale. Les droits pourraient être des " rappels pour nous aider à nous souvenir des relations morales fortes " qui font

${ }^{18}$ Cité par John Witte \& Latterell, Justin J., " Christianity and Human Rights : past Contributions and Future Challenges ", The Journal of Law and Religion; Atlanta Vol. 30, Iss. 3, (Oct 2015) : 353-385.

${ }^{19}$ Cité par John Witte \& Latterell, Justin J., "Christianity and Human Rights : past Contributions and Future Challenges ", The Journal of Law and Religion; Atlanta Vol. 30, Iss. 3, (Oct 2015) 
la vie chrétienne. Mais les chrétiens feraient bien de se débarrasser du vocabulaire des droits moraux en soi, par crainte de dévaloriser leur discours moral ou d'amoindrir leur moralité. ${ }^{20}$

Ceci n'est qu'une brève évocation de deux exemples d'oppositions chrétiennes aux droits de l'homme qui développent un argumentaire critique à partir de la plus grande exigence de la morale chrétienne, comparée à cela, la morale des droits serait une petite morale de compromis dont les dits droits sont remplis d'évidences morales qu'il parait indécent de rappeler.

A contrario, le théologien étasunien John Witte, dans son grand ouvrage, Religious Human Rights in Global Perspectives, ${ }^{21}$ note les risques d'une dépréciation des droits de l'homme, y compris dans leurs dimensions religieuses. Cette critique a des effets néfastes.

1) D'abord parce que les droits de l'homme ont leur racine dans les droits à la liberté de conscience et d'opinion (Révolution français et Américaines) Cf Thèse de Georg Jellinek. ${ }^{22}$

2) Cette dépréciation divise plus encore le monde occidental du monde non-occidental sur la validité des droits de l'homme,

3) Enfin cette dépréciation exagère le rôle des états dans la garantie des droits. Notamment Hauerwas ignore des trois types et trois générations de droits : la première génération, les droit civils et politiques, la deuxième génération, les droits culturels et économiques et enfin la troisième génération, les droits écologiques.

J'ajouterai deux remarques à celles de John Witte. La première est la notion de droits. En effet, celle-ci n'est pas acquise et ceci pour deux raisons, l'une linguistique l'autre proprement juridique. La DUDH ne comporte pas d'impératif mais est écrite au présent, un présent de

${ }^{20}$ Simone Weil, L'enracinement, ou prélude à une déclaration des devoirs envers l'être humains, Paris, Champs, classiques, 2014.

${ }^{21}$ John Witte, Johan D. van der Vyver, Religious Human Rights in Global Perspectives, Emory University, 1996.

${ }^{22}$ Georg JELLINEK, La déclaration des droits de l'homme et du citoyen : contribution à l'étude du droit constitutionnel moderne, traduction de l'allemand, Paris, Fontemoing,1902. 
description, ${ }^{23}$ une sorte d'a priori, "qui se situe avant toute chose ", mais dont il se pourrait, et ceci est vérifiable dans bien des pays, que l'on ne fasse rien. D'un point de vue juridique, la DUDH n'est pas un texte de loi. Chaque pays est tenu de traduire dans sa constitution les articles de la déclaration. A ce moment-là seulement, la déclaration, qui alors n'en est plus une, devient un texte de loi. La seconde est à proprement parler théologique. Nous n'avons pas besoin de la DUDH pour savoir que la torture est un crime, dit Hauerwas. Il me semble que cet argument est un peu hasardeux, qu'il ignore l'histoire chrétienne et par conséquent l'historicité de la foi et la maturation possible des doctrines chrétiennes et qu'il fait fi d'un principe de hiérarchie des vérités, enfermé sans doute dans une théologie communautariste où la singularité de la communauté d'appartenance est d'emblée plus vraie et plus juste tandis que la rencontre de l'altérité religieuse et philosophique altère et ternit la profondeur de la foi. Il est symptomatique de voir comment Simone Weil et Stanley Hauerwas évitent la question de l'universalité. A leur décharge, il faut dire que la notion d'universalité ou d'universel comporte des ambigüités et des malentendus. Comme l'explique le philosophe François Jullien, l'universel européen a une triple origine qui ne parvient pas à s'unifier ${ }^{24}$, entre son origine grecque, scientifique et philosophique à partir du concept nécessairement vrai pour tous, son origine romaine juridique pour tous les citoyens romains, urbs et orbis et enfin, l'origine chrétienne liée à l'universalité du salut en Jésus-Christ où dans la foi il n'y a plus ni grec ni juif, ni esclave ni homme libre. ${ }^{25}$ A cela s'ajoute une confusion contemporaine entre l'universel, l'uniforme et le commun qui ont besoin d'être réinterrogés. En effet, l'universel confondu d'emblée soit avec le général soit avec une nécessité qui s'impose, se révèle aujourd'hui être une singularité occidentale ${ }^{26}$. La question de l'être, de la substance par exemple, est peu traduisible dans un autre espace culturel et dans des langues qui ne

${ }^{23}$ QUELQUEJEU Bernard, "De quelle universalité les droits de I'homme relèvent-ils ? ", dans Revues des sciences philosophiques et théologiques, Vrin, 2011/3, tome 95, p.619-630.

${ }^{24}$ François JULLIEN, II n'y a pas d'identité culturelle, mais nous défondons des ressources d'une culture, Paris, Editions de L'Herne, 2017, chapitre 2.

${ }^{25}$ Gal 3, 28

${ }^{26}$ François JULLIEN, Ibid. chapitre 1. 
sont pas indo-européennes. D’autre part notre environnement culturel a tendance à confondre l'universel et l'uniforme. L'uniforme est commercial et commode, il n'est que la répétition de l'un sans attention possible au sujet. Enfin, le commun est trop souvent confondu avec le semblable. Or, explique Jullien le bien commun est une rencontre inclusive d'altérités, c'est le semblable qui vire au communautarisme. Contrairement, à Simone Weil et Stanley Hauerwas il apparaît que l'exigence la plus haute est du côté de la construction d'un universel comme rencontre des altérités, un universel ouvert, un universel des possibles. Si le christianisme est avant tout une foi traversée par la rencontre de l'autre/Autre (Une altération dit le théologien Joseph Caillot) alors la plus grande exigence morale est là dans la tâche de bâtir de l'universel au nom même de l'universalité du salut. La théologie chrétienne ne peut abandonner l'universel car cela touche le cœur de sa Révélation, portée dans les vases d'argiles de nos singularités culturelles : c'est donc bien cela son exigence première.

Y-a-t-il une évidence que la torture soit un crime ? C'est bien méconnaitre notre histoire du christianisme que de dire cela. Combien d'hommes ou de femmes ont succombé sous les tortures de la Sainte inquisition ? Il ne s'agissait pas d'accidents de l'histoire vite condamnés par le Magistère, il s'est agit de pratiques régulières et normées affectant des soi-disant hérétiques ou de soi-disant sorcières. Bien d'autres exemples historiques seraient à étudier de plus prêt. L'esclavage est un autre exemple instructif. Combien de temps les Eglises ont-elles mis pour condamner l'esclavage ? Pourtant la lecture de Saint Paul ne souffre pas d'ambigüité s'il n'y a ni esclave ni homme libre, on pourrait imaginer qu'il faille en déduire immédiatement à l'égalité entre tous les hommes, une égale dignité de tous les êtres humains et pas seulement les chrétiens. Ainsi, il est important pour notre propos de reconnaitre que l'histoire de l'Eglise est ainsi faite que la vie de foi purifie sa raison théologique, affine sa conscience au cours de son histoire. "Si nous adoptons une approche plus historique de la raison et reconnaissons son développement dans l'enseignement social de l'Église elle-même, alors, nous pouvons reconnaitre que l'enseignement social de l'Église est le résultat de la purification de la raison et de la formation des consciences. La foi purifie la raison, clarifie la conscience, et a permis de mener à bien le développement 
de l'enseignement social de l'Eglise. » explique le théologien australien Neil Ormerod. ${ }^{27}$ Ce dernier reprend dans des catégories historiques les notions traditionnelles de nature et grâce, à partir du binôme " creating and healing ${ }^{28}$, c'est-à-dire créativité et guérison qui caractérise un double mouvement lié à la nature historique de la vie de foi de l'Eglise. Il y a donc un mouvement vers le haut, "creative vector » où l'intelligence pratique de l'homme crée de nouvelles situations, nouvelles technologies, nouvelles politiques économiques et sociales selon une échelle de valeurs vitales (scale vital values ${ }^{29}$ ). Ce processus créatif a besoin d'être soutenu par des valeurs culturelles qui lui donnent sens. Ceci est un mouvement lié à la nature historique de l'homme. Mais d'un autre côté, ce mouvement créatif doit être intégré à un mouvement de guérison (healing) du haut vers le bas, où la grâce vient interpeller et soigner les distorsions dans le processus créatif pratique de la condition historique de l'homme. Dans ce mouvement, il note; "Religious value (grace) heals the distortions of consciousness... and cultural values and also... challenge for social values " ${ }^{30}$. Avec le "creative vector", Neil Ormerod ajoute donc le « healing vector". Son échelle de valeur est faite pour être empruntées du bas vers le haut autant que du haut vers le bas. Ce double mouvement, inséparable, selon notre auteur caractérise la condition historique de la vie de foi de l'Eglise. ${ }^{31}$ Ce double mouvement de créativité historique et sociale et de guérison spirituelle et théologique est bien à l'œuvre dans cette histoire complexe des droits de l'homme et permet de penser les évolutions de la doctrine non pas comme des trahisons mais comme des imbrications de la foi dans l'histoire et comme l'élaboration d'une intelligence de la foi plus ajustée à la vie chrétienne selon l’Evangile.

${ }^{27}$ Ouvrage collectif, Neil J. Ormerod, Shane Clifton, Identity and Mission in Catholic Agencies, St Paul éditions, 2009, p.67-82,

${ }^{28}$ Neil Ormerod, op.cit. p.76. Ces catégories sont explicitement empruntées à Bernard Lonergan.

${ }^{29}$ Ibid. p.76

${ }^{30}$ Ibid. p.76-77.

31 Ibid. p.77, "just as the creative process, when unaccompanied by healing, is distorted and corrupted by bias, so too the healing process, when unaccompanied by creating, is a soul without a body". Citation de Lonergan. 


\section{Construire de l'universel à partir des singularités historiques}

Nous sommes bien aujourd'hui devant le défi de penser l'universalité des droits de l'homme avec la reconnaissance de la pluralité des fondements philosophiques ou religieux. Dit autrement, l'humanisme est pluriel, déjà en occident, mais encore plus dans le monde et c'est donc une impasse d'en chercher le fondement philosophique exclusif. En réalité, la déclaration elle-même est un a priori auquel différents systèmes de pensée essaient de s'adapter. L'exemple du Magistère de l'Eglise catholique est typique de cette adaptation comme nous l'avons vu. Après avoir rejeté violemment les droits de l'homme tout au long du XIX è siècle, l'Église catholique depuis Jean XXIII, y a consenti et de façon active depuis 40 ans.

Cette histoire des droits de l'homme n'obéit donc pas à un principe dont il faudrait faire l'application. Au contraire, cette histoire nous montre une manière historique de penser et de construire de l'universel qui ne découle pas d'un concept nécessaire et intemporel depuis les grecs. Ensuite cette histoire des droits de l'homme et de la déclaration de 1948 nous incite à penser la construction de l'universel à partir de ce qui se vit, s'expérimente, se vérifie dans les cultures, les pays et les communautés humaines. Nous en avons déjà des exemples. Celui de la non-violence de Gandhi et de Martin Luther King est significatif. En effet l'action politique de Gandhi s'est appuyée sur les principes indouistes de l'Ahimsa, développée par des réflexions sur le droit (l'expérience de l'Afrique du sud) enrichi par la lecture d'Henri-David Thoreau et son traité sur la désobéissance civile ${ }^{32}$ mais aussi par la lecture de l'Evangile et l'invitation à aimer ses ennemis. ${ }^{33}$ Quand Martin Luther King se met à lire Gandhi, il comprit en interprétant et en adaptant les principes d'actions de la Nonviolence l'intérêt fondamental pour la lutte pour les droits civiques en s'apercevant que Gandhi lui-même a puisé chez Henri-David Thoreau la réflexion sur la désobéissance civile. L'Ahimsa non-violence, est devenue par son voyage interculturel un objet universalisable dont il serait vain

32 Henri-David Thoreau, La désobéissance civil, Paris, Mille et une nuit, 1997.

33 Mahatma Gandhi, Tous les hommes sont frères, Paris, Folio/essais Gallimard, 1969.

Rev. Pistis Prax., Teol. Pastor., Curitiba, v. 11, n. 1, 008-028, jan./abr. 2019 
de limiter la compréhension à une origine exclusive. En effet, c'est à cette source déjà plurielle que Don Helder Camara puisa l'inspiration pour son action de lutte pour la justice auprès des pauvres du Brésil. Dans son petit ouvrage Spirale de Violence, l'archevêque de Récif retravailla l'héritage de Gandhi et de Luther-King ${ }^{34}$ avec une analyse sociale de la violence, de ses causes et des ces enchainements. A partir de cette analyse, l'action non-violence libératrice devient un art de ne pas entrer dans la spirale de la violence et ses trois niveaux d'enchainement. ${ }^{35}$ La non-violence est donc réinterprétée et resituée par Helder Camara dans le contexte social et politique brésilien. C'est la circulation et l'interprétation en contexte de ce bien culturel reconnu pour sa fécondité, qui en fait un universel, pourtant non achevé. Et c'est parce qu'elle continuera à circuler à travers les cultures et les contextes politiques et sociaux que la non-violence deviendra un bien universel non saturé. Un bien commun culturel en migration.

\section{Conclusion}

\section{La théologie confrontée aux rapports entre pluralité, singularité et universalité}

A la fin de ce parcours historique et théologique il nous est apparu clairement que l'universalité des droits de l'homme ne pouvait s'imposer comme une sorte d'impératif catégorique au risque de comprendre cette universalité et ces droits de l'homme comme une singularité occidentale des Lumières pensant s'imposer à tous par un jeu de concepts érigés en un contexte historique qui n'a pas l'évidence d'être de tous. Cette contestation des droits de l'homme ne se fait pas toujours en raison, elle abrite parfois le profit de tyrans ou de chefs religieux intégristes. Il faut donc être attentif à des questionnements qui permettent d'étendre ou de penser de meilleurs manières les droits de l'homme aujourd'hui et de les

\footnotetext{
${ }^{34}$ Le livre est dédié à la mémoire de Gandhi et de Luther King.

35 Don Helder Camara, Spirale de violence, Paris, Desclée de Brouwer, 1970.
} 
distinguer des remises en cause qui sont la justification de dictatures de toutes sortes.

Il nous est apparu également que la recherche des fondements idéologiques des droits de l'homme n'a pas permis une synthèse. Osons le dire, quand une religion, une philosophie ou une nation essaie de s'attribuer une origine principale aux droits de l'homme, c'est une impasse. Cette investigation de la bonne origine a rencontré une pluralité de perspectives, souvent objets de polémiques scientifiques ou idéologiques et pas toujours indemne d'autojustification. Ce fut la tentative, réussie pour une part de la DUDH de 1948 d'écrire une déclaration qui, au-delà des interprétations des déclarations américaines et françaises de la fin du XVIII ${ }^{\text {è }}$ siècle, ne justifierait aucun parti pris et n'adopterait aucune transcendance métaphysique fondatrice particulière même si la tentation y était réellement présente dans la tâche justificatrice confiée à l'UNESCO après la seconde guerre mondiale comme nous l'avons vu. Si choisir un fondement était impossible, la synthèse heureuse des fondements est apparue est aussi impossible.

L'évolution de la position du Magistère catholique sur les droits de l'homme nous a d'abord surpris par sa radicalité, d'une condamnation sans appel au XIX ̀̀ siècle par Grégoire XVI à un fondement du bien commun chez Jean-Paul II le gap paraissait infranchissable. Cette évolution est pourtant très instructive sur l'historicité de l'expérience de la foi et partant du murissement et de la maturité de la foi de l'Eglise. Celle-ci est sans conteste un objet théologique propre, entre créativité historique et soin par la foi. L'autre dimension du débat contemporain sur les droits de l'homme est la possibilité ou non d'une universalité. Dans un monde éclaté, où les communautarismes semblent gagner la partie, peut-on et désire-t-on encore penser et construire de l'universel ? Au débat classique entre singularité/particularité et universalité s'ajoute aujourd'hui la pluralité radicale des options, des philosophies, des cultures, des religions. Il s'agit donc d'une triade : singularité, universalité et pluralité dont l'historicité est certaine et la recherche d'un bien commun nécessaire. Les théologiens ne peuvent être indifférents à ce débat dans l'espace public. Ils doivent s'y engager parce qu'est en jeu ce qui touche au cœur la Révélation 
chrétienne, l'historicité et l'universalité du salut au milieu de la pluralité contemporaine des conceptions de l'homme.

\section{Références}

ALBERIGO, G. Histoire du Concile Vatican II, 1959-1965 : Tome IV - l'Eglise en tant que communion. Louvain; Paris : Éditions du Cerf ; Peeters, 2003.

CÂMARA, H. Spirale de violence. Paris : Desclée de Brouwer, 1970.

CONCILE VATICAN II. Dignitatis humanae [DH 1965]. Disponible en : <http:// www.vatican.va/archive/hist_councils/ii_vatican_council/documents/vat-ii_ decl_19651207_dignitatis-humanae_fr.html>. Consulté en : 06 Janvier, 2019.

CONGAR, Y. Que faut-il entendre par déclaration?. In : Vatican II, la liberté religieuse. Paris : Éditions du Cerf, 1967, p. 47-52 (Colléction Unam Santam).

GANDHI, M. Tous les hommes sont frères. Paris : Folio; Gallimard, 1969.

GREGOIRE XVI. Mirari vos [MV 1832]. Disponible en : <http://jesusmarie.free. fr/encyclique_mirari_vos.html>. Consulté en : 04 Janvier, 2019.

JEAN XXIII. Pacem in terris: Lettre encyclique sur la paix entre toutes les nations, fondée sur la vérité, la justice, la charité et la liberté [PT 1963]. Disposnible en : <http://w2.vatican.va/content/john-xxiii/fr/encyclicals.index.html〉. Consulté en 04 Janvier, 2019.

JELLINEK, G. La déclaration des droits de l'homme et du citoyen : contribution à l'étude du droit constitutionnel moderne. Paris : Fontemoing,1902.

JOAS, H. Comment la personne est devenue sacrée, nouvelle généalogie des droits de l'homme. Genève : Labor et Fides, 2015.

JULLIEN, J. Il n'y a pas d'identité culturelle, mais nous défondons des ressources d'une culture. Paris : Éditions de L'Herne, 2017.

MARITAIN, J. Introduction à la Déclaration des Droits de l'Homme. Paris : Éd. UNESCO, 1948. Spécialement le texte PHS/3 rev. 
ORMEROD, N. J. ; SHANE, C. Identity and Mission in Catholic Agencies. London : St Pauls, 2009.

QUELQUEJEU, B. De quelle universalité les droits de l'homme relèvent-ils ? In: Revue des Sciences Philosophiques et Théologiques, tome 95, n. 3 / 2011, Vrin, p. 619-630.

THOREAU, H. D. La désobéissance civil. Paris : Mille et une nuits, 1997.

WEIL, S. L'enracinement, ou prélude à une déclaration des devoirs envers l'être humains. Paris : Champs Classiques, 2014

WITTE, J. ; LATTERELL, J. Christianity and Human Rights : past Contributions and Future Challenges. In : The Journal of Law and Religion, Atlanta, vol. 30, Iss. 3, Octobre de 2015, p. 353-385.

WITTE, J. ; VAN DER VYVER, J. Religious Human Rights in Global Perspectives. Atlanta : Emory University, 1996.

ZUBER, V. L'origine religieuse des droits de l'homme, le christianisme face aux libertés modernes (XVIII'̀-XXI ̀̀ siècle). Genève : Labor et Fides, 2017.

Reçu : 13/03/2019

Aprovado : 13/03/2019

Approuvé : 17/04/2019

Aprovado : 17/04/2019 